This pre-print refers to the paper presented in IEEE EEEIC 2019 (Genoa, 11-14 June 2019), with DOI:10.1109/EEEIC.2019.8783587

\title{
Remote Hardware-In-the-Loop Measurement System for Electrolyser Characterization ${ }^{1}$
}

\author{
Andrea Mazza, Abouzar Estebsari, \\ Giulia Morandi, Ettore Bompard \\ Dip. Energia "Galileo Ferraris" Politecnico di Torino \\ Torino, Italy \\ \{andrea.mazza, abouzar.estebsari, ettore.bompard\}@polito.it
}

\author{
Harm Lok \\ Centre of Expertise Energy Entrance \\ Hanze University of Applied Sciences \\ Groningen, The Netherlands \\ h.lok@pl.hanze.nl
}

\begin{abstract}
The installation of facilities replicating the real-world condition is often required for carrying out meaningful tests on new devices and for collecting data with the aim to create realistic device model. However, these facilities require huge investments, as well as areas where they can be properly installed. In this paper, we present a test infrastructure exploiting the concept of Remote Power Hardware-In-the-Loop (RPHIL), applied for characterizing the performances of a $8 \mathrm{~kW}$ Proton Exchange Membrane (PEM) electrolyser installed at the Hanze University of Applied Sciences in Groningen (The Netherlands). The electrolyser is subjected to different test conditions imposed both locally and remotely. The results show that this measurement procedure is effective and can open new perspectives in the way to share and exploit the existing research infrastructure in Europe.
\end{abstract}

Keywords: Real Time Simulation, Remote Power Hardware-in-theloop, PEM electrolyser, measurements, performance tests.

\section{Introduction}

The introduction of new Renewable Energy Sources (RES) posed new challenging to the electricity system and led researches to study the best way to solve these new issues, for example by introducing virtual inertia [1] [2], reconfiguration procedures [3] (both single objective [4] and multi-objective

\footnotetext{
${ }^{1}$ This contribution has received funding from the European Union's Horizon 2020 research and innovation programme under grant agreement No. 691797 (project STORE\&GO). The paper only reflects the authors' views and the European Union is not liable for any use that may be made of the information contained herein.
} 
This pre-print refers to the paper presented in IEEE EEEIC 2019 (Genoa, 11-14 June 2019), with DOI:10.1109/EEEIC.2019.8783587

[5]) or storage facilities [6] [7]. Currently, two main paradigms are competing for the future shape of the electrical system: the supergrid paradigm [8], and the microgrids paradigm [9]. In the first case, the system will be reinforced through a massive expansion of the transmission system, while in the latter case the system will be based on autonomous communities. In both cases, new tools are necessary to investigate the complexity of the entire system. One of the possible answers to this lies in the share of already existing research infrastructures, by putting together different laboratories. These kinds of projects exploit the potentiality of the Real Time Simulation (RTS) and allow to properly implementing the concept of "laboratory in the network" [10], [11]. An example of this kind of infrastructure is the ERICLab [12], making possible co-simulation among laboratories in Politecnico di Torino (Italy), RWTH Aachen University (Germany), JRC Petten (The Netherlands) and JRC Ispra (Italy). Another example successfully implemented is the RT-Superlab, which put together European and American research institutions for making possible a unique real-time transatlantic co-simulation [13]. All the above examples was inspiring the possibility to use this kind of approach also in the project STORE\&GO [14]. This project aims to investigate the role of Power-to-Gas (PtG) for the energy transition, and to exploit it as a long-term storage able to accommodate the excess of RES. In this framework, the properly integration of this technology into the electrical system is requested, for understanding the best potential applications and how to exploit this technology as better as possible (in terms, for example, of dynamic performances) [15]. This paper aims to present the use of the connection established between Politecnico di Torino and Hanze University of Applied Sciences for making remotely tests in a $8 \mathrm{~kW}$ Proton Exchange Membrane (PEM) electrolyser. The use of this connection i) allows to have direct access to the facilities located in Hanze from the researchers at Politecnico (reaching the goal of sharing of research facilities throughout Europe) and ii) is the first step towards a co-simulation involving Remote Power Hardware-In-the-Loop (RPHIL) with a use of a power amplifiers supplying the electrolysers and set remotely. The paper is composed as follows: Section II explains the different types of co-simulation existing and how including PHIL in it. Section III shows the facilities of the Hanze university. In Section IV are described the test cases and the results, while Section V contains the Conclusions. 


\section{Explanation of the remote hardware in the loop infrastructure}

\subsection{Co-simulation and Hardawe-in-the-Loop}

To test and validate the performance of a device prototype or to tune or characterize a newly manufactured hardware before installing in the system (e.g. connecting to power grid), a so-called hardware in-the-loop is often carried out using digital real-time simulators.

In cases, the device is a source of energy (e.g. solar panel, wind turbine connected generator, fuel cell, etc.) or sink of energy (i.e. loads), an interface is needed to enable power exchange. In such cases, a power amplifier is exploited to perform a Power Hardware In-the-Loop (PHIL). The power interface is the key element in PHIL experiments. The power interface is made of a power amplifier and a set of sensors which monitor the hardware under test (HUT). The power amplifier acts as a source or sinks in order to respectively generate or absorb power. From the simulated grid it is possible to extract the values of voltage or current to control the output of the power amplifier in order to feed the HUT.

Multidisciplinary analysis is needed to ensure the right performance of a newly developed technology (e.g. prosumer, micro-grid controller, electrolyser, etc.) in the overall system. This directly introduces new challenges to the hardware in-the-loop experiments due to the following reasons:

- New devices or technologies require interdisciplinary studies among different expertise areas;

- Interoperability analysis of newly integrated technology is crucial;

- The rest of system (i.e. simulated model of the grid) could be a largescale energy system;

- The rest of system may be a "system of systems" with variant geographical and energy behavioral (e.g due to social or cultural habits) characteristics;

The remote hardware-in-the-loop measurement system for global that we established for prosumer characterization is based on the so-called "geographically distributed real-time co-simulation" [10] [16].

Although the vision of this approach is not limited to only remote laboratory (Hardware/Software) access, but "Internet-Distributed Hardware In-the-Loop (HIL)" has attracted many researchers since last twenty years. We intend to implement and use such an internet-distributed HIL in two steps: firstly to enable a laboratory (PoliTo's) to access Real-Time Target of the other laboratory (Hanze's), load Simulink models through Internet, and run a PHIL remotely provided the hardware set-up is done (Figure 1 
This pre-print refers to the paper presented in IEEE EEEIC 2019 (Genoa, 11-14 June 2019), with DOI:10.1109/EEEIC.2019.8783587

(B)); the next phase is to decouple the "rest of system" (i.e. virtual model of the grid and behavioral scenarios) to be concurrently run on simulators located in both distant laboratories (Figure 1(C)).

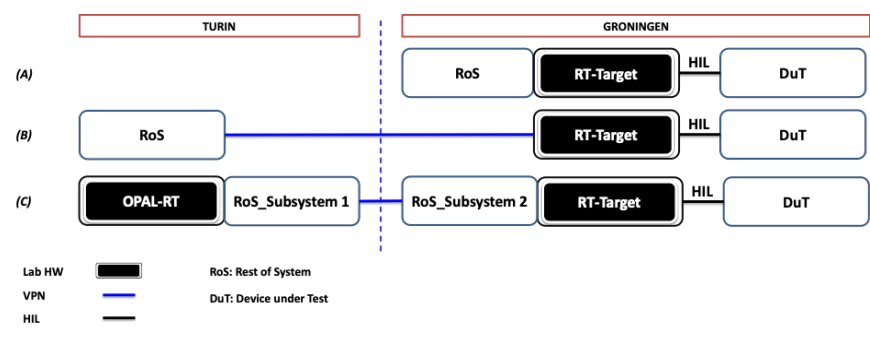

Figure 1: General overview of planned PHIL between PoliTo and Hanze.

Figure 1 depicts a very high level general scheme of PHIL collaboration between PoliTo and Hanze, eventually realizing an internet-distributed HIL, and providing a multi-site laboratory test set-up for prosumer and electrolyser characterization and grid impact analysis.

\section{Network connection and Co-simulation}

Figure 2 shows the two physically separated local area networks (LANs), one placed in Groningen and the other one in Turin. They are merged into a single segment via a layer 2 Ethernet bridge connection. In the configuration created in this test bed, Groningen is the main LAN; there is created a virtual hub on the OpenVpn server and a local bridge connection is realized to the network adapter on the specific LAN. The side of Turin represents the sub-LAN; in that side a PC connected to the Opal/RT LAN is equipped with a virtual OpenVpn server and hub which is bridged to the LAN connection. When a connection is initiated from Turin, a cascade connection is made between Turin and Groningen. At this moment, the two separated network segments are converted into a single segment, whose two sides can communicate between each other. This fact leads to have a connection between devices which seems to operate in the same network segment. So every side setup can be configured as both operate in the same LAN.

\section{Description of the facilities in Hanze}

\subsection{Overview of the Hanze setup}

The real-time set-up is installed at the Hanze's Entrance test site in Groningen (The Netherlands). This hardware setup aims to test the integration of $\mathrm{P} 2 \mathrm{G}$ into the electrical grid, which involves the characterization of various new connected entities that can be composed of different sources and 


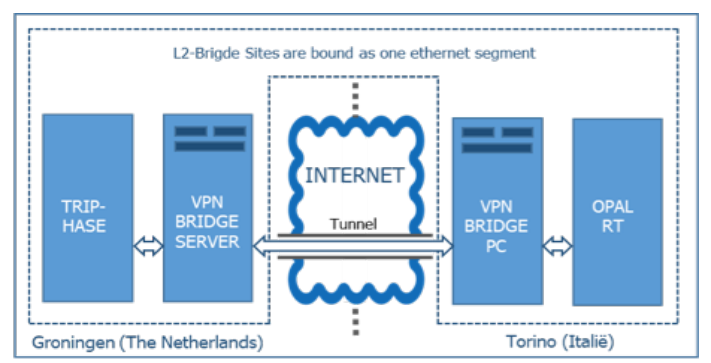

Figure 2: The Layer 2 Ethernet Bridge Connection

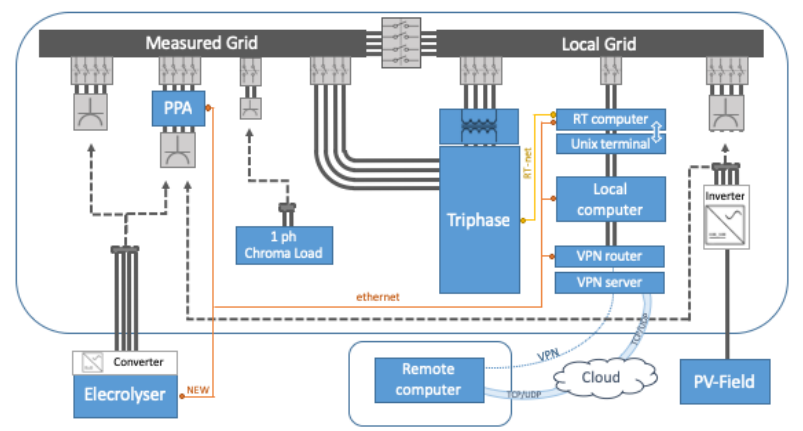

Figure 3: The real-time micro grid setup installed in Hanze

loads (e.g., combination of wind turbines, PV panels, fuel cells, electrolysers and others). The various subsystems and both the physical and virtual connections between them are schematized in Figure 3.

\subsection{Real-time measured-grid}

By referring to Figure 2, one can see that the real-time measured-grid is independent and galvanic separated from the local grid. The grid is constructed as three phases with neutral conductor and is functionally equivalent to the most common European 3-phase AC networks. The setup also includes the possibility to connect a single phase electronic load. This could be switched to either of the three phases and create a programmed load pattern to test individual phase reactions or achieve a predefined unbalance in the three phases.

\subsection{Power Amplifier}

The creation of a controllable grid (in terms of both voltage and frequency) needs a power amplifier (PA) which is able to replicate in the grid the requested condition. The PA in the test-bed consists of a $15 \mathrm{~kW}$ bidirectional isolated power module with 6-channel output, suited for bidirectional energy 
flow between the supply grid and single or three-phase AC or DC loads and sources. For virtual operation, the PA power module is connected through a real-time communication bus (called "XC real-time network") to a powerful PC-based real-time control unit. This PC is able to run the models for all kind of customized sources or loads in real-time (e.g., the PA can be configured as either voltage source or current source, depending on the customer choice).

\subsection{Virtual connection}

The communication network of the real-time grid is based on a router directly connected to the internet backbone of Hanze's datacenter, providing a high-speed $1 \mathrm{~Gb} / \mathrm{s}$ Ethernet connection. This connection encompasses the following devices: local engineering PC, real-time PC and power analyser. Each device is provided of its own IP-address. In this way, the installed devices can be real-time monitored by the power analyzer and accessed by the local computer via the TCP/IP connection.

\subsection{PV field}

The PV-field consists of 16 panels with a total nominal power of $4.4 \mathrm{kWp}$ The generated DC power is transformed into AC by a three-phase inverter, which, as the electrolyser, can be connected to the measured internal grid through the power analyzer, so that the power production and all the related electrical variables are monitored and logged. Otherwise, by connecting the inverter to the local external grid, the power production is sent directly back into the electrical network.

\subsection{Electrolyser}

The electrolyser connected is a fully automated ${ }^{2} \mathrm{PEM}$ ) based water electrolysis system of $8 \mathrm{~kW}\left(1 \mathrm{Nm}^{3}\right.$ hydrogen per hour). It is designed for indoor operation only, thus is located in a container that consists of two separated rooms. One of them contains the PLC and the water preparation system (where tap water is treated by reverse osmosis and ion exchange filters to create suitable water for electrolysis), whereas the other room contains the "electrolyser system", composed of:

- an electrolysis cell stack

\footnotetext{
${ }^{2}$ According to the manual this means that the operator only has to start the system up at the beginning and shut it down at the end of the operation, while all the internal variable control (e.g., for safety purposes) is made automatically by the electrolyser control system
} 


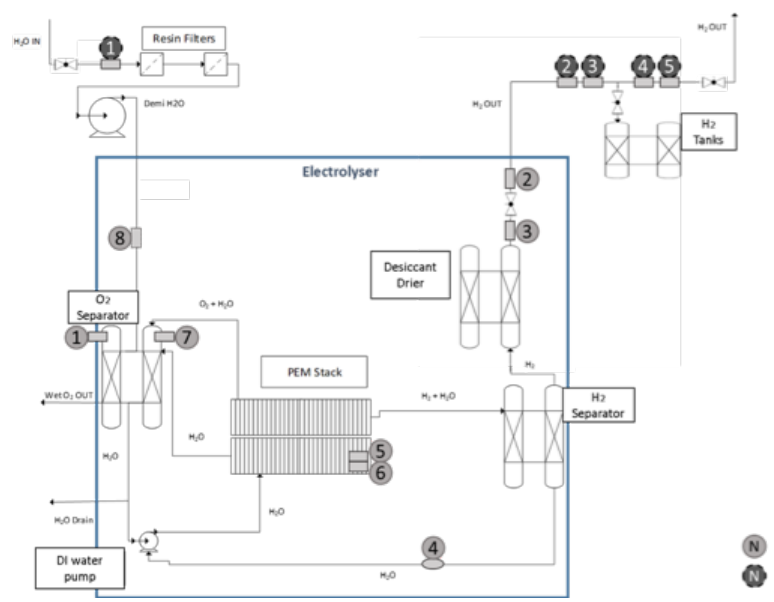

Figure 4: Process flow diagram of the electrolyser

- auxiliary equipments, necessary for regulating electrolysis operations (circulating water, drying hydrogen, pressurizing hydrogen and shutting down the system)

- sensors and control board for monitoring performance

Furthermore, in the same room where the electrolyser is located, a system of valves and two 5-litre tanks for storing the produced hydrogen is also installed. The tanks can be emptied afterwards by venting the contained hydrogen outside of the unit. Produced hydrogen can also be directly vented during operation, without filling the tanks. Figure 4 shows the process flow diagram of the hydrogen production and the location the different above components inside the unit.

The electrolyser has the following operating states:

- Pre-Start: The state begins when the main power switch is closed. When Pre-Start operation is completed, it is possible to maintain this idle state or moving into the next state.

- Generate-Vent: In this mode, the circulation pump is started up and the system verifies the process values of water flow rate and water quality. Full current is applied to the stack after 120 seconds and hydrogen generation is started. The system checks the levels of both separator levels (i.e., $\mathrm{H}_{2}$ /water and $\mathrm{O}_{2}$ /water), voltage conditions in the stack and rectifier operation, and vents hydrogen and oxygen outside during this operation. If the process values pass all checkpoints, the electrolyser will stop venting and start to build up system pressure, leading to next state. 
This pre-print refers to the paper presented in IEEE EEEIC 2019 (Genoa, 11-14 June 2019), with DOI:10.1109/EEEIC.2019.8783587

- Pressurize Storage: In this state, the hydrogen circuit in the system is pressurized up to the pressure regulator set-point (approximately 15 bar). Once reached this pressure value, the process goes into steadystate.

- Steady-State: The electrolyser produces hydrogen that flows intermittently out of the system because the hydrogen is produced only when the $\mathrm{H}_{2}$ pressure in the tank is below the set point. Once the set-point is reached, the electrolyser stops the production of hydrogen, remaining in hot stand-by. In case of no-tank, the hydrogen production continues indefinitely.

\subsection{Flow controller}

A flow controller has been recently added on the output product pipe and its communication channel has been connected to the laboratory LAN. In this way, the instrument allows both to control and measure the amount of produced gas flowing through it and all its data and settings can be accessed through Modbus TCP/IP protocol by a computer connected to the same LAN (being directly or via VPN, as for the other instruments already installed). In particular, this device has been installed for the purpose of allowing studies on the electrolyser dynamic behaviour. In fact, the hydrogen production rate can be considered roughly proportional to the electrical current of the stack and this affecting the overall (AC) electrical consumption of the unit. It follows a step change in the set-point of hydrogen output flow produces a sudden change of electrical power consumption of the entire system.

\subsection{Measure of electrolyser-related variables}

The values related to the hydrogen production and the system variables (e.g., water inlet) can be monitored and logged in two separated ways, i.e., by downloading from the PLC located in the container, or directly from the electrolyser through the use of a commercial software via RS-232 connection. With reference to Figure 4, Table 1 and Table 2 show the measurement devices in the system, their location in the process flow diagram and whether their data can be accessed either from the PLC side or from the electrolyser side.

\subsection{Measure of electrical variables}

Besides the mentioned sensors and measurement instrumentation included inside the electrolyser container, the laboratory is equipped with a registered and calibrated power analyser. The analyzer samples the voltage and current wave forms entering or exiting the prosumer, within a typical sampling time 
Table 1: PLC monitored variables

\begin{tabular}{|c|l|l|}
\hline & \multicolumn{2}{|c|}{ PLC monitored variables } \\
\hline 1 & $\mathrm{H}_{2} \mathrm{O}$ inlet volume flow & {$[\mathrm{ml} / \mathrm{min}]$} \\
\hline 2 & Produced $\mathrm{H}_{2}$ volume flow (upstream of the tanks) & {$\left[\mathrm{m}^{3} / \mathrm{h}\right]$} \\
\hline 3 & Produced $\mathrm{H}_{2}$ pressure (upstream of the tanks) & {$[\mathrm{bar}]$} \\
\hline 4 & $\begin{array}{l}\text { Produced } \mathrm{H}_{2} \text { volume flow (downstream of the } \\
\text { tanks) }\end{array}$ & {$\left[\mathrm{m}^{3} / \mathrm{h}\right]$} \\
\hline 5 & Produced $\mathrm{H}_{2}$ pressure (downstream of the tanks) & {$[\mathrm{bar}]$} \\
\hline
\end{tabular}

Table 2: HOGEN Monitored Variables

\begin{tabular}{|r|l|l|}
\hline & \multicolumn{2}{|c|}{ HOGEN monitored variables } \\
\hline 1 & Gas detect & {$[\% \mathrm{LeL}]$} \\
\hline 2 & Product pressure & {$[\mathrm{psi}]$} \\
\hline 3 & System pressure & {$[\mathrm{psi}]$} \\
\hline 4 & Water quality & {$[\mathrm{M} \quad \square]$} \\
\hline 5 & Stack voltage & {$[\mathrm{V}]$} \\
\hline 6 & Stack current & {$[\mathrm{A}]$} \\
\hline 7 & System temperature & {$\left[{ }^{\circ} \mathrm{C}\right]$} \\
\hline 8 & Water flow rate & {$[\mathrm{lpm}]$} \\
\hline *Other variables related to the converter monitoring such as: \\
3.3 Vdc sense, 24.0 Vdc sense, +5.0 Vdc sense, $2.5 \mathrm{~V}$ reference, -5.0 \\
Vdc sense, board temperature, spare thermistor \\
\hline
\end{tabular}




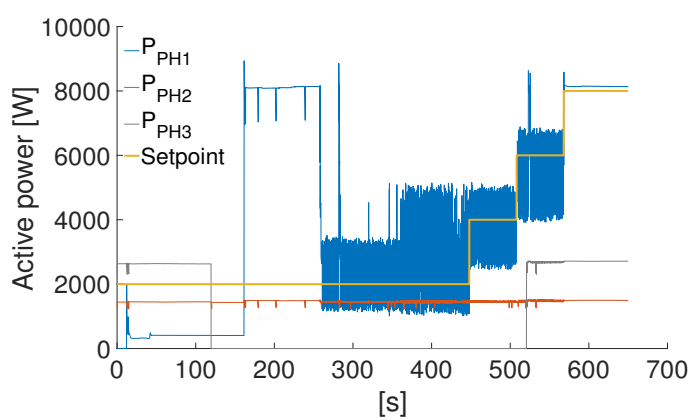

Figure 5: Active power withdrawn by the electrolyser - Local measurements

of $0.5 \mu \mathrm{s}$. Once sampled, the data is to be processed by FPGA or DSPs and the various derived parameters are calculated within the same time frame. Another important dynamic feature (necessary for accurate power measurements), is that the selected power analyzer is able to record, store, process and delete samples "on the fly". This means that there is never a gap in the sampling process, since the memory buffer is continually emptied after the samples are processed.

\section{$5 \quad$ Test Cases and Results}

Thanks to the installed devices, multiple tests were performed, presented in the next subsections.

\section{$5.1 \quad$ First test set}

The first experimental set was conducted by logging data through the use of the software programs provided with the measuring devices. In this way, it was possible to set the sample time of both the instruments to the lowest value that they are capable to handle. Figure 5 illustrate the active power for all the three phases and measurements performed locally in Groningen, whereas Figures 6 shows the same parameters with data logging carried out from Turin.

The set-point given (manually) to the flow controller is the same for both cases: the electrolyser is started up with the flow controller set to $25 \%$ of maximum flow rate and this value is kept constant for 7 minutes to make sure that the electrolyser has been correctly started and the steady state has been reached. Then, three subsequent steps of 1 minute duration increase the set-point of $25 \%$ at each time (1 minute at 50\%, 1 minute at $75 \%$ and finally 1 minute at $100 \%$ of the maximum flow rate). From both Figure 5 and 6 it is evident the phase imbalance due to the supply of the DC rectifier mainly through a single phase (i.e., phase 1 ). The phase 1 power values follow the operating states of the electrolyser: at the first, 


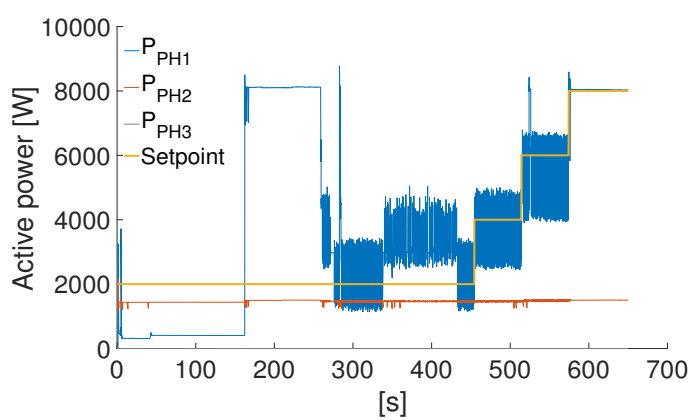

Figure 6: Active power withdrawn by the electrolyser - RHIL measurements

the electrolyser withdraws low power (around $400 \mathrm{~W}$ ) corresponding to the generating-vent mode. Then, full power is applied to the stack in order to build up system pressure in correspondence of the pressurise storage state. Afterwards, the electrolyser operates in steady state at four different power (i.e., $25 \%, 50 \%, 75 \%, 100 \%$ of the nominal power). In correspondence to the three first set-points, the actual power varies within a wide range around a mean value roughly equal to them. On the other hand, at full load the power is approximately constant and equal to the maximum value of $8 \mathrm{~kW}$. The wide jumps at reduced load are due to the way in which the control of the unit is built. Basically, the supply of the stack is switched on and off in order to maintain the system pressure around the set-point. Comparing the data acquired from Groningen and Turin, some differences can be observed due to the fact that the electrolyser behavior is not perfectly reproducible (e.g., the generating-vent mode can last longer depending on the initial water quality, the waveform representing the power consumption at a certain time can be shifted depending on the switching control initial instant and so on). It follows that the delay due to the data transmission via VPN between the two laboratories cannot be inferred from a comparison of the two graphs. However, it has been possible to obtain some information about it by looking deeply into the packets exchange. The lowest sample time handled by the power analyzer is $20 \mathrm{~ms}$ and it is respected at each time step when the measurements are performed from Groningen. From Turin instead, the power analyzer sample time of $20 \mathrm{~ms}$ is respected only on average, while at each time step a packet can be received either early or late because of packets queuing due to the communication latency.

\subsection{Second test set}

The second series of experiments had the aim of carrying out the data monitoring and logging from within a MATLAB Simulink environment, in order to make it possible to subsequently integrate the hydrogen production unit into network models on the basis of RHIL concepts. The model is composed 
of three different conceptual parts:

- Flow controller Modbus writing

- Flow controller Modbus reading

- Power analyzer TCP reading

Each part contains a MATLAB function implemented intentionally either for writing or reading the respective instrument, taking into account the protocol requirements and also the necessary translation (may be ASCII, binary, decimal or hexadecimal). The results obtained by giving the same setpoints of the first set of tests are shown in Figures 7 (model run from Groningen) and 8 (same model run from Turin).

All the considerations presented in Section 5.1 are still valid here. The main difference highlighted in these cases is that the ranges of power variation are consistently reduced. In fact, now both the instruments are read with a sample time roughly equal to $400 \mathrm{~ms}$ from Groningen and 600 from Turin. It follows that the measured values at each sample time is just an average of the data samples within the considered interval. This is caused by the introduction of a certain latency in the communication between MATLAB Simulink the instruments, as well as by the MATLAB Simulink functioning. If the simulation is performed as an off-line simulation, the sample time of the instruments within the model is constrained by the simulation calculation time. On the other hand, if the simulation is run in real-time, the simulation time step itself is constrained by the minimum sample time that the instruments are able to handle. Finally, the hydrogen output flow measured through a dedicated software in Groningen (lower sample time) is shown in Figure 9 whereas Figure 10 presents the hydrogen output measured through the Simulink model (higher sample time). Besides the huge difference in time steps, the two graphs are barely distinguishable, since the dynamic of the hydrogen flow lasts no more than 1 second.

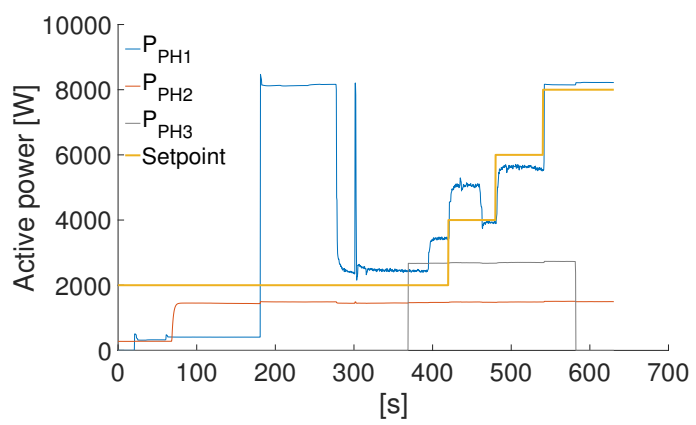

Figure 7: Active power withdrawn by the electrolyser - Local measurements 


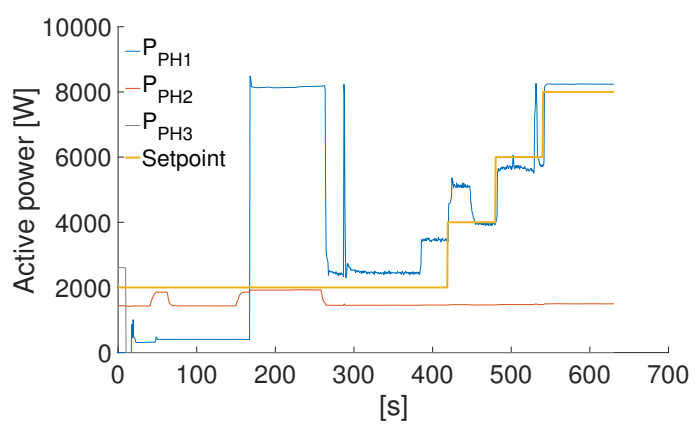

Figure 8: Active power withdrawn by the electrolyser - RHIL measurements

\section{Conclusions}

The paper shows the application of the concept of RHIL for characterizing the electrical behavior of the electrolyser. This characterization is necessary because the large deployment of $\mathrm{P} 2 \mathrm{G}$ plants needs studies regarding the dynamic of the electrolyser, both for properly coupling with the RES plants and with the rest of the $\mathrm{P} 2 \mathrm{G}$ plant chain $\left(\mathrm{H}_{2}\right.$ storage and methanation unit). The additional value provided by the RHIL concept lies into the possibility to get the information regarding the electrical behavior of the HUT remotely, and directly integrating it into a simulated environment replicating the real network conditions. The presence of the entire measurement chain introduces delays, and from the tests shown in Section 5.1 was evident that the imposed sample time $(20 \mathrm{~ms})$ can be only be respected in average, whereas by imposing directly the flow rate through the Simulink model (i.e., Section 5.2) the sample time increases from 400 to $600 \mathrm{~ms}$, with an additional time equal to $200 \mathrm{~ms}$ for remote measurement with respect to the local measurement. Nevertheless, even with the above mentioned limitation, the measurement layout seems to be feasible for research purposes, especially because allows to access to facilities throughout Europe, by enhancing the collaboration between different institution. The next steps will

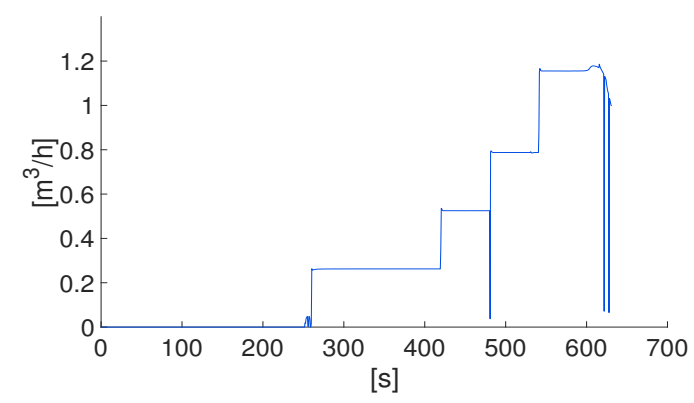

Figure 9: Production of $\mathrm{H}_{2}$ - Local measurements

978-1-7281-0653-3/19/\$31.00 C 2019 IEEE 


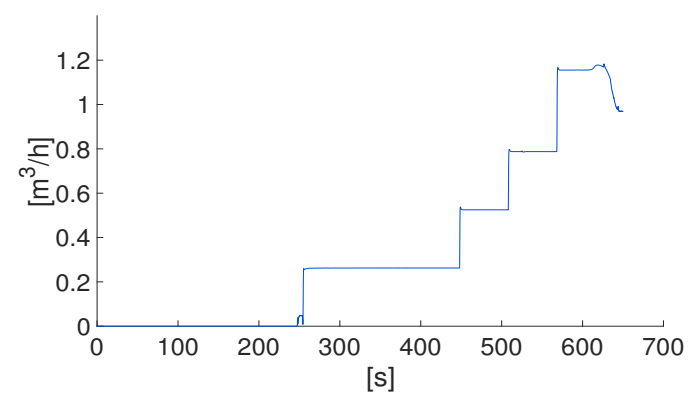

Figure 10: Production of $\mathrm{H}_{2}$ - RHIL measurements

be to integrate directly the electrolyser into a network simulation through the power amplifiers, for studying the response of the electrolyser to electrical signal (both in normal and abnormal network conditions), by collecting remotely at the same time all the electrolyser variables.

\section{References}

[1] H. Bevrani, T. Ise, and Y. Miura, "Virtual synchronous generators: A survey and new perspectives," International Journal of Electrical Power \& Energy Systems, vol. 54, pp. 244 - 254, 2014.

[2] S. Rubino, A. Mazza, G. Chicco, and M. Pastorelli, "Advanced control of inverter-interfaced generation behaving as a virtual synchronous generator," in 2015 IEEE Eindhoven PowerTech, pp. 1-6, June 2015.

[3] G. Chicco and A. Mazza, "An overview of the probability-based methods for optimal electrical distribution system reconfiguration," in 2013 4 th International Symposium on Electrical and Electronics Engineering (ISEEE), pp. 1-10, Oct 2013.

[4] A. Merlin and H. Back, "Search for a minimal loss operating spanning tree configuration in an urban power distribution system," in 5th power system computational conference, pp. 1-6, October 1975.

[5] A. Mazza, G. Chicco, and M. Rubino, "Multi-objective distribution system optimization assisted by analytic hierarchy process," in 2012 IEEE International Energy Conference and Exhibition, ENERGYCON 2012, pp. 1-6, Sep 2012.

[6] M. Aneke and M. Wang, "Energy storage technologies and real life applications a state of the art review," Applied Energy, vol. 179, pp. 350 $-377,2016$. 
This pre-print refers to the paper presented in IEEE EEEIC 2019 (Genoa, 11-14 June 2019), with DOI:10.1109/EEEIC.2019.8783587

[7] H. Blanco and A. Faaij, "A review at the role of storage in energy systems with a focus on power to gas and long-term storage," Renewable and Sustainable Energy Reviews, vol. 81, pp. 1049 - 1086, 2018.

[8] F. Schettler, M. Balavoine, M. Callavik, J. Corbett, N. Kuljaca, V. Larsen, N. MacLeod, and B. Sonerud, "Roadmap to the supergrid technologies," Friends of the SuperGrid, 2012.

[9] R. Lasseter, A. Akhil, C. Marnay, J. Stephens, J. Dagle, R. Guttromson, A. S. Meliopoulous, R. Yinger, and J. Eto, "Integration of distributed energy resources. the certs microgrid concept," 2002.

[10] C. F. Covrig, G. De Santi, G. Fulli, M. Masera, M. Olariaga, E. Bompard, G. Chicco, A. Estebsari, T. Huang, E. Pons, et al., "A european platform for distributed real time modelling \& simulation of emerging electricity systems," JRC Report, JRC101772, EUR 27941 EN, 2016.

[11] M. Stevic, S. Vogel, M. Grigull, A. Monti, A. Estebsari, E. Pons, T. Huang, and E. Bompard, "Virtual integration of laboratories over long distance for real-time co-simulation of power systems," in IECON 2016 - 42nd Annual Conference of the IEEE Industrial Electronics Society, IEEE, October 2016.

[12] "Eric-Lab Website." Available: https://www.eric-lab.eu/.

[13] A. Monti, M. Stevic, S. Vogel, R. W. D. Doncker, E. Bompard, A. Estebsari, F. Profumo, R. Hovsapian, M. Mohanpurkar, J. D. Flicker, V. Gevorgian, S. Suryanarayanan, A. K. Srivastava, and A. Benigni, "A global real-time superlab: Enabling high penetration of power electronics in the electric grid," IEEE Power Electronics Magazine, vol. 5, pp. 35-44, September 2018.

[14] "STORE\&GO website." Available: https://www.storeandgo.info/.

[15] A. Mazza, E. Bompard, and G. Chicco, "Applications of power to gas technologies in emerging electrical systems," Renewable and Sustainable Energy Reviews, vol. 92, pp. $794-806,2018$.

[16] E. Bompard, A. Monti, A. Tenconi, A. Estebsari, T. Huang, E. Pons, M. Stevic, S. Vaschetto, and S. Vogel, "A multi-site real-time cosimulation platform for the testing of control strategies of distributed storage and v2g in distribution networks," in 2016 18th European Conference on Power Electronics and Applications (EPE'16 ECCE Europe), pp. 1-9, September 2016. 